\title{
Application of Ultrafast Schottky Diodes to High Megahertz Chaotic Oscillators
}

\author{
G. Mykolaitis ${ }^{a, b}$, A. Tamaševičius ${ }^{a, *}$, S. Bumelient $\dot{B}^{a}$, \\ A. NAmajūnas ${ }^{a}$, K. Pyragas ${ }^{a}$ And V. Pyragas ${ }^{a}$ \\ ${ }^{a}$ Semiconductor Physics Institute, A. Goštauto 11, Vilnius 01108, Lithuania \\ ${ }^{b}$ Department of Physics, Faculty of Fundamental Sciences
}

Vilnius Gediminas Technical University, Saulètekio 11, Vilnius 10223, Lithuania

\begin{abstract}
The considered chaotic oscillator consists of an amplifier, 2nd order LC resonator, Schottky diode and an extra capacitor in parallel to the diode. The diode plays the role of a nonlinear device. Chaotic oscillations are demonstrated numerically and experimentally at low as well as at high megahertz frequencies, up to $250 \mathrm{MHz}$.
\end{abstract}

PACS numbers: 05.45.Ac, 05.45.Pq, 05.45.- a, 47.52.+j

\section{Introduction}

The Schottky-barrier diodes are commonly classified as low-noise high-speed semiconductor devices for high frequency and microwave applications. When reverse-biased at the breakdown voltages they can be employed as noise sources characterized by an effective noise temperature from $10^{3} \mathrm{~K}$ to $10^{4} \mathrm{~K}[1]$.

In the present paper we describe high-power noise generator with a Schottky diode. Actually it is a dynamical noise (chaos) generator similar to the tunnel diode oscillator driven by external sinusoidal voltage $[2,3]$. The main shortcoming of the externally driven (non-autonomous) systems is that sharp peaks do appear in the power spectra at the drive frequency and its higher harmonics. In contrast, the dynamical noise generator suggested in this paper is an autonomous one, providing essentially smoother power spectra. Two experimental prototypes of the oscillator operating at different fundamental frequencies $f^{*}=1 / 2 \pi \sqrt{L C}$ have been investigated. The first one has been set at low megahertz frequency $\left(f^{*} \approx 1 \mathrm{MHz}\right)$, meanwhile the second one has been tuned to operate at high megahertz frequency $\left(f^{*} \approx 100 \mathrm{MHz}\right)$.

${ }^{*}$ corresponding author 


\section{Structure of the dynamical noise generator}

A simplified diagram of the dynamical noise generator is shown in Fig. 1. A series 2nd-order LC resonator and a non-inverting amplifier are common building

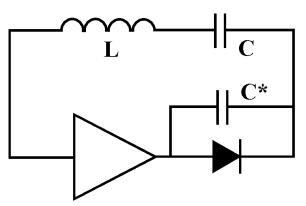

Fig. 1. Circuit diagram of chaotic oscillator with the Schottky diode. DC bias circuit is not shown for simplicity.

blocks of a classical sinusoidal oscillator. However, additional elements, namely the Schottky-barrier diode and an extra capacitor $C^{*}$ are inserted in the positive feedback loop. The diode plays the role of a nonlinear device, while the capacitor $C^{*}$ acts as an extra energy storage (inertial) element. The overall system becomes a nonlinear dynamical system with three degrees of freedom, thus it is a potentially chaotic oscillator [4].

\section{Equations and numerical results}

Dynamics of the oscillator is given by the following rate equations:

$$
\begin{aligned}
& C \dot{V}_{C}=I_{L}, \\
& L \dot{I}_{L}=\left(k R_{\text {in }}-R_{\text {in }}-R_{\text {out }}\right) I_{L}-V_{C}-V_{d}, \\
& C^{*} \dot{V}_{d}=I_{0}+I_{L}-I_{\mathrm{S}}\left(\exp \frac{q V_{d}}{k_{\mathrm{B}} T}-1\right) .
\end{aligned}
$$

Here $V_{C}$ and $I_{L}$ is the voltage across the capacitor and the current through the inductor of the LC resonator, respectively; $V_{d}, I_{0}$, and $I_{\mathrm{S}}$ is the voltage, the dc bias current, and the reverse saturation current of the Schottky diode, respectively; $k, R_{\text {in }}$, and $R_{\text {out }}$ is the gain, the input and the output resistance of the amplifier, respectively. The exponential term in the third equation represents the currentvoltage characteristic of the diode. By introducing the dimensionless variables and parameters

$$
\begin{aligned}
& x=\frac{V_{C}}{V_{T}}, \quad y=\frac{\rho I_{L}}{V_{T}}, \quad z=\frac{V_{d}}{V_{T}}, \quad t^{\prime}=\frac{t}{\tau}, \\
& V_{T}=\frac{k_{\mathrm{B}} T}{q}, \quad \rho=\sqrt{\frac{L}{C}}, \quad \tau=\sqrt{L C}, \\
& a=\frac{R_{\mathrm{n}}}{\rho}(k-1)-\frac{R_{\mathrm{out}}}{\rho}, \quad b=\frac{\rho I_{0}}{V_{T}}, \quad c=\frac{\rho I_{\mathrm{S}}}{V_{T}}, \quad \varepsilon=\frac{C^{*}}{C},
\end{aligned}
$$

the following set of equations convenient for numerical integration is obtained: 
$\dot{x}=y$,

$\dot{y}=a y-x-z$,

$\varepsilon \dot{z}=b+y-c(\exp z-1)$.
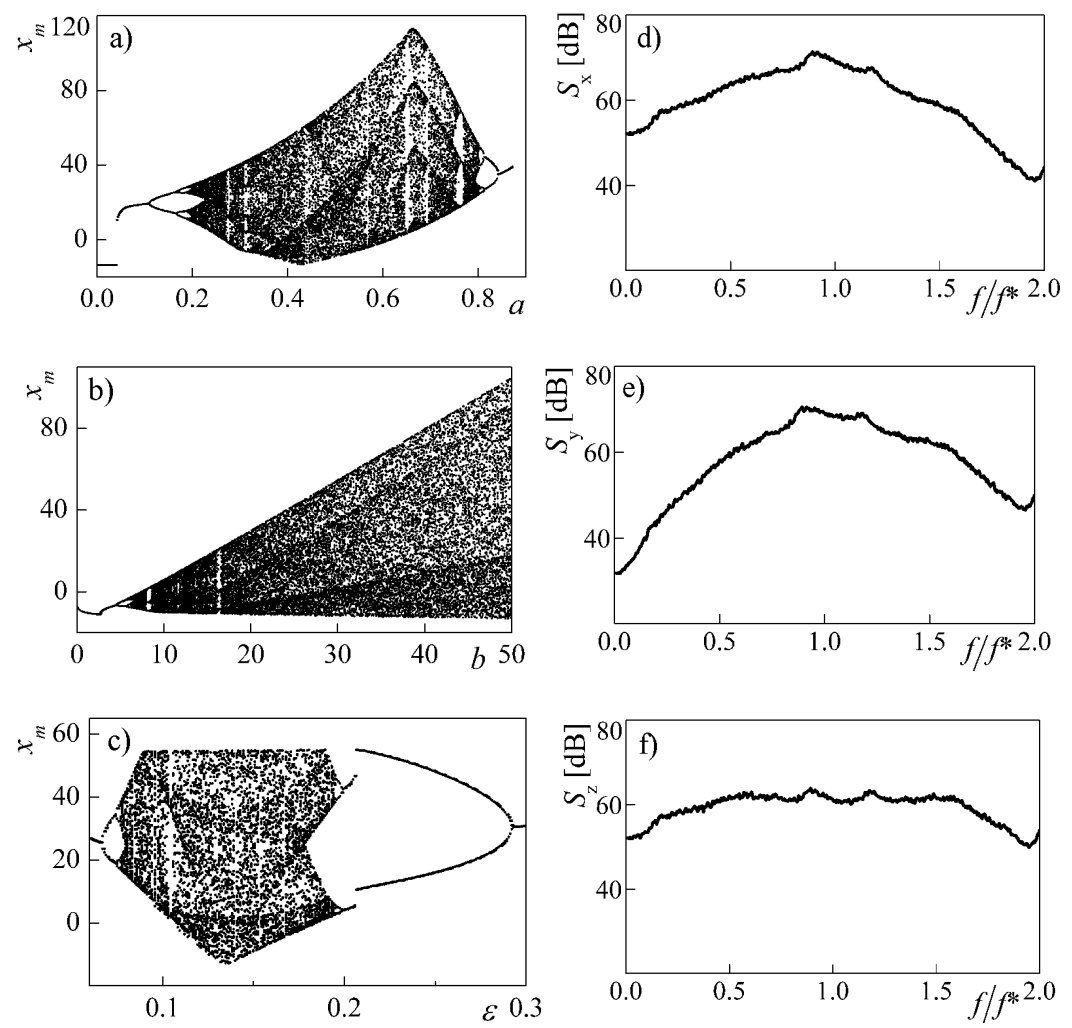

Fig. 2. Left part: bifurcation diagrams, i.e. local maxima $x_{\mathrm{m}}$ of variable $x(t)$ versus control parameters $a, b$, and $\varepsilon$ at $c=4 \times 10^{-5}$ : (a) $b=30, \varepsilon=0.13$; (b) $a=0.4$, $\varepsilon=0.13$; (c) $a=0.4, b=30$. Right part: power spectra $\left(f^{*}=1 / 2 \pi \tau\right)$ calculated from variables $x, y$, and $z$ at $a=0.4, b=40, \varepsilon=0.13$.

The oscillator exhibits period-doubling route to chaos, narrow periodic windows in the chaotic domains, and reverse bifurcations as illustrated with the bifurcation diagrams in Fig. 2. Several power spectra are also presented.

\section{Experimental results}

Experiments were carried out using silicon Schottky diodes, namely BAT41 or BAT43 (recovery time $\tau_{\text {rec }}=5 \mathrm{~ns}$, junction capacitance $C_{d}=2 \mathrm{pF}$ ) at low 

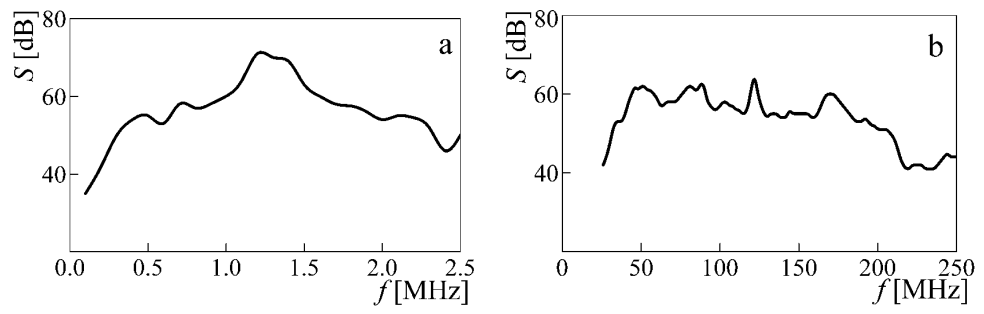

Fig. 3. Power spectra taken at the output $\left(V_{\text {out }}=k R_{\text {in }} I_{L} \propto y\right)$ of the amplifier for different diodes. (a) BAT41, $I_{0} \approx 7 \mathrm{~mA}, L=8 \mu \mathrm{H}, C=2.7 \mathrm{nF}, C^{*}=360 \mathrm{pF}$, $k \approx 2, R_{\text {in }}=50 \Omega, R_{\text {out }} \approx 0$. Resolution $\Delta f=70 \mathrm{kHz}$. (b) HSMS8101, $I_{0} \approx 3 \mathrm{~mA}$, $L=100 \mathrm{nH}, C=22 \mathrm{pF}, C^{*}=3.3 \mathrm{pF}, k \approx 3, R_{\text {in }}=R_{\text {out }}=50 \Omega$. Resolution $\Delta f=120 \mathrm{kHz}$. The level of $0 \mathrm{~dB}$ corresponds to effective noise temperature of $10^{5} \mathrm{~K}$.

megahertz frequency and microwave diode $\operatorname{HSMS} 8101\left(\tau_{\mathrm{rec}}=25 \mathrm{ps}, C_{d}=0.3 \mathrm{pF}\right)$ at high megahertz frequency. The results are presented in Fig. 3 as broadband continuous power spectra. These spectra are in a qualitative agreement with the numerical ones.

\section{Conclusions}

Schottky diodes have been demonstrated to be applicable to high megahertz frequency chaotic oscillators. The effective noise temperature in the frequency range from 50 to $200 \mathrm{MHz}$ is about $10^{11} \mathrm{~K}$, i.e. 7 orders higher than in [1]. In addition, the power spectrum is essentially smoother than of the tunnel diode based oscillator $[2,3]$. Schottky diode chaotic oscillators potentially can be set to gigahertz frequencies by removing the capacitor $C^{*}$ (its role plays the junction capacitance $C_{d}$ ) and setting the LC resonator elements at $L \leq 10 \mathrm{nH}$ and $C \leq 2 \mathrm{pF}$.

\section{Acknowledgment}

G.M., A.T., and S.B. were supported in part by Lithuanian State Science and Studies Foundation under contract No. T-62/04.

\section{References}

[1] L.P. Dunleavy, J. Randa, D.K. Walker, R. Billinger, J. Rice, IEEE Trans. Microwave Theory Techn. 46, 2620 (1998).

[2] A. Namajūnas, A. Tamaševičius, Elektron. Tekhn. Ser. 2 1, 69 (1990) (in Russian).

[3] A. Namajūnas, A. Tamaševičius, Liet. fiz. rink. 32, suppl., 85 (1992).

[4] E. Ott, Chaos in Dynamical Systems, Cambridge University Press, Cambridge 1993. 